\title{
Fluorescence Lifetimes and Emission Patterns Probe the 3D Orientation of the Emitting Chromophore in a Multichromophoric System
}

\author{
Wouter Schroeyers, ${ }^{\dagger}$ Renaud Vallee,${ }^{\dagger}$ Digambara Patra, ${ }^{\ddagger}$ Johan Hofkens, ${ }^{*}, \dagger$ Satoshi Habuchi, ${ }^{\dagger}$ \\ Tom Vosch, ${ }^{\dagger}$ Mircea Cotlet, ${ }^{\dagger}$ Klaus Müllen, ${ }^{\#}$ Jörg Enderlein,, ${ }^{*}$ and Frans C. De Schryver, ${ }^{\star}$, \\ Department of Chemistry, Katholieke Universiteit Leuven, Celestijnenlaan 200F, 3001 Heverlee, Belgium, \\ Max-Planck-Institut für Polymerforschung, Ackermannweg 10, 55128 Mainz, Germany, and \\ Institute for Biological Information Processing I, Forschungszentrum, D-52425 Jülich, Germany
}

Received April 30,2004; E-mail: johan.hofkens@chem.kuleuven.ac.be; j.enderlein@fz-juelich.de; Frans.DeSchryver@chem.kuleuven.ac.be

\section{(W) This paper contains enhanced objects available on the Internet at http://pubs.acs.org/journals/jacsat.}

The optoelectronic properties of dyes embedded in thin polymer films are currently under intense study due to the use of such materials in nanotechnology ${ }^{1}$ and nano-electronics. ${ }^{2}$ The fluorescence lifetime of a single fluorescent molecule (SM) embedded in a thin film can fluctuate as a direct manifestation of the SM nanoenvironment (e.g., as a result of polymer dynamics). ${ }^{3-5}$ Furthermore, it has been shown that the fluorescence lifetime strongly depends on the position and orientation of the SM with respect to the boundaries of the film, an effect known as the influence of the electromagnetic boundary conditions (EBC) ${ }^{6-13}$ This effect is especially important in very thin polymer films $(<30$ $\mathrm{nm})$. Therefore, several groups have devoted efforts to determine the $3 \mathrm{D}$ orientation of a SM in thin polymer films. ${ }^{14-17}$ Techniques such as annular illumination (probing the orientation of the absorption transition dipole) ) $^{15,16}$ and wide-field defocusing (probing the emission transition dipole moment) have been proposed and exploited. ${ }^{17}$

In this Communication, we report on the fluorescence behavior of a single first generation multichromophoric dendrimer with four perylene imide chromophores at the rim (G1R4) embedded in a thin polymer film (Chart 1). ${ }^{18,19}$ A model developed to describe energy transfer in this system ${ }^{13}$ invokes energy hopping between the different chromophores. It was suggested that, at any moment in time, the emission of a single dendrimer originates from the chromophore that has the lowest energy (fluorescent trapping site) due to the inhomogeneous nature of the polymer matrix used for immobilization. ${ }^{20}$ Due to polymer dynamics and/or consecutive photobleaching, each of the chromophores in the dendrimer can in time become the fluorescent trapping site. Since the chromophores in the dendrimer have a well-defined 3D orientation with respect to each other (tetrahedral for G1R4), probing the dipole orientation of the emitting chromophore of a single G1R4 molecule using the two above-mentioned complementary observables, namely emission patterns and the EBC effect on the fluorescence lifetime, should allow us to validate the hopping model.

To do so, G1R4 dendrimers at single-molecule concentration were embedded in thin $(10-25 \mathrm{~nm})$ zeonex (poly(norbornene)) films. To probe the orientation of the emitting chromophore emission dipole, we used defocused $(1 \mu \mathrm{m}$ defocusing distance with respect to the glass - polymer interface) wide-field imaging. ${ }^{6,17}$ On the basis of the characteristic intensity distribution of the defocused images, the determination of the emission dipole orientation

\footnotetext{
$\dagger$ Katholieke Universiteit Leuven.

¥ Max-Planck-Institut für Polymerforschung.

\# Institute for Biological Information Processing I.
}

Chart 1. Dendrimer with Four Perylenene Imide (PI) Chromophores

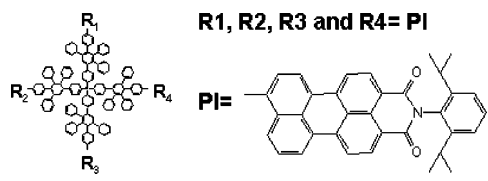

becomes feasible. Böhmer et al. ${ }^{17}$ provided exact wave-optical calculations of these defocused images. Upon comparing the experimental data with calculated results, we obtained the emission dipole orientation.

The patterns shown in Figure 1 were observed sequentially during the indicated period. One sees that the emission pattern of a single G1R4 molecule changes as function of time. The patterns provide direct evidence that different chromophores of the G1R4 SM emit in time, as different dipole orientations are observed successively. In this series we observe only three different patterns, probably one chromophore bleached during alignment. On the basis of the simulated patterns (Figure 1b), ${ }^{6,21}$ following the sequence shown in Figure 1a, we attribute the first and second patterns to a chromophore with an emission dipole oriented in-plane with angles of $40^{\circ}$ and $70^{\circ}$, respectively, with respect to the $x$-axis $\left(\theta=90^{\circ}\right.$, $\phi=40^{\circ}$ and $\left.\theta=90^{\circ}, \phi=70^{\circ}\right)$. The third pattern indicates an out-of-plane oriented chromophore $\left(\theta=0^{\circ}, \phi=0^{\circ}\right)$. Note that the error for the out-of-plane orientation $\theta$ is $\pm 15^{\circ}$, whereas a very high accuracy in the $x y$ plane can be obtained..$^{21}$

Since the emission patterns give direct evidence for the hopping model between the tetrahedral oriented chromophores in individual G1R4 molecules, the EBC effect for a SM of G1R4 should result in discrete changes in the fluorescence lifetime. By using a scanning confocal optical microscope with polarization-sensitive FIFO mode detection, the intensity, the degree of polarization $P$, and the fluorescence lifetime of a G1R4 SM were simultaneously determined. ${ }^{6}$

Figure 2 shows the intensity recorded in the two orthogonal polarization channels (a), the degree of polarization (b), and the fluorescence lifetime (c) trajectories of a single G1R4. Each emissive level clearly corresponds to a different polarization value. The four different emissive levels are thus related to four different emitting chromophores. The lifetime trajectory shows an increase of the fluorescence lifetime from $4.8 \mathrm{~ns}$ for the highest emissive level to approximately $7 \mathrm{~ns}$ for the other three lower emissive levels. Such an anticorrelated behavior between the lifetime and the intensity can be attributed to the EBC effect. Indeed, the emission of a chromophore oriented more or less in the plane of the sample 

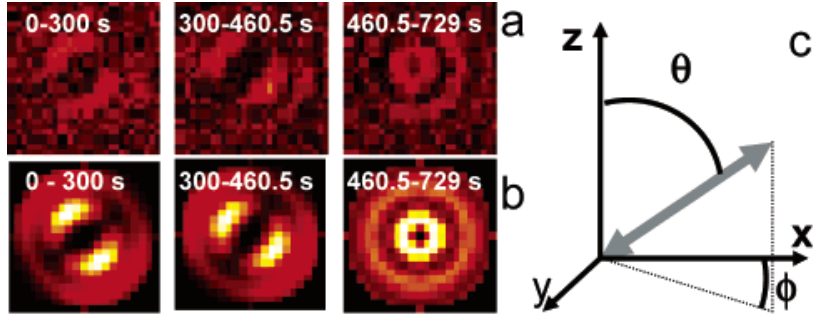

Figure 1. Emission patterns recorded for a single G1R4 molecule in a 25 $\mathrm{nm}$ zeonex film at $1 \mu \mathrm{m}$, defocusing to the glass surface and at $0.5 \mathrm{~s}$ exposure time (a). The depicted images are binned 10 times. By means of pattern recognition software ${ }^{21}$ the experimental patterns were compared with simulated patterns (b). (c) Definition of angles: $\theta$ is the inclination angle between the optical dipole (double arrow in gray) and optical axis ( $z$-axis), and $\phi$ indicates the in-plane (in the $x y$ plane) dipole orientation.

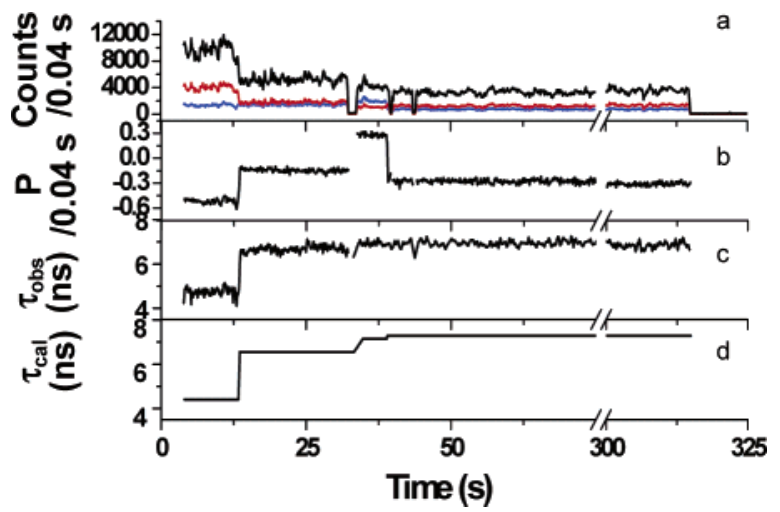

Figure 2. Fluorescence intensity (counts) (a), polarization $(P)(b)$, and lifetime $\left(\tau_{\text {obs }}\right)(\mathrm{c})$ trajectories for a single G1R4 dendrimer embedded in a $10 \mathrm{~nm}$ thick zeonex film. In part a, the blue and red traces correspond to the intensity trajectories in the two orthogonal polarization channels. A simulated fluorescence lifetime trace $\left(\tau_{\mathrm{cal}}\right)(\mathrm{d})$ was calculated for a dendrimer with location $(z) 4 \mathrm{~nm}$ under the glass surface. ${ }^{6}$

is expected to give a shorter lifetime and higher emissive rate than a chromophore oriented more perpendicular with respect to the air interface. ${ }^{8}$ The absence of jumps in the simultaneously recorded emission spectra ${ }^{6}$ ensures furthermore that the lifetime jumps are not connected to other processes in the matrix. As mentioned earlier, the structure of a G1R4 SM in a polymer film resembles a tetrahedron. The four chromophores of the system can thus be placed in such a way that three of them are similarly oriented more perpendicular, relative to the interface, while in comparison the fourth chromophore is oriented more parallel. Figure $2 \mathrm{~d}$ shows a simulation of the lifetime change of G1R4 for such an orientation, ${ }^{6}$ clearly confirming this assumption. Both imaging and lifetime measurements confirm hopping to a fluorescent trap in G1R4.

For the investigated molecules (158), 40\% show EBC-type lifetime fluctuations. The largest observed lifetime fluctuation in a $10 \mathrm{~nm}$ film is from 4 to $12 \mathrm{~ns}$, in good agreement with the calculated lifetimes obtained for parallel and perpendicular oriented chromophores. ${ }^{6}$ An important parameter related to the hopping model presented for G1R4 is the relative inhomogeneous character of the environment acting on the SM. In such a situation, each chromophore feels a slightly different environment. In contrast, for a more homogeneous local environment, the energies of the four chromophores are nearly identical. In the latter case, each individual chromophore has now a probability to emit. Due to the finite integration time, the detected signal then results in an averaging of the fluorescence of the different chromophores. An example is given in Figure 3. For the series of emission patterns depicted in Figure $3 \mathrm{a}$, the $3 \mathrm{D}$ orientations of three patterns could be assigned on the basis of simulated patterns (Figure 3b) as $\theta=90^{\circ}, \phi=0^{\circ}$ for the

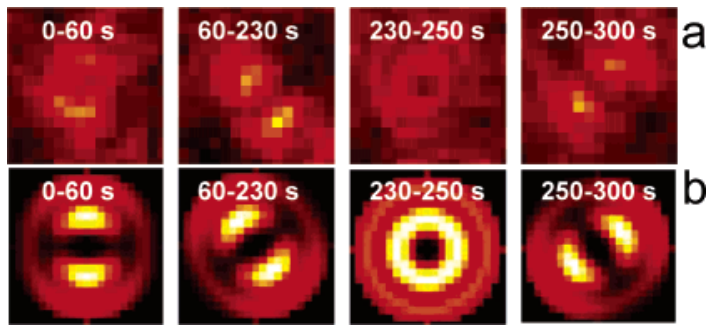

Figure 3. Series of emission patterns (a) and corresponding simulated patterns (b) for a single G1R4 molecule in a $25 \mathrm{~nm}$ zeonex film. Images were recorded at $1 \mu \mathrm{m}$ defocusing and $10 \mathrm{~s}$ exposure time. ${ }^{21}$

(N) A movie of the emission patterns series shown in part a is available. first pattern, $\theta=90^{\circ}, \phi=45^{\circ}$ for the second pattern, and $\theta=$ $90^{\circ}, \phi=135^{\circ}$ for the fourth pattern.

The third pattern, however, according to the calculations, is a combination of patterns, indicating multiple emitters in this time interval. Notice the different features between the third patterns of Figures $3 \mathrm{~b}$ and $1 \mathrm{~b}$, indicating multiple emitting chromophores (average pattern) and an out-of-plane oriented chromophore, respectively. ${ }^{6}$ The switching in time between distinct patterns related to chromophores acting as fluorescent traps and the average pattern (even at $0.5 \mathrm{~s}$ time resolution) indicates that the homogeneity of the polymer surrounding this particular molecule fluctuates as a result of polymer dynamics. A more detailed study of the influence of local polymer inhomogeneity will be published elsewhere.

Acknowledgment. R.V. and S.H. thank the FWO and the JSPS, respectively, for a postdoctoral fellowship. The KULeuven research fund, the Federal Science Policy through IAP/V/03, the Flemish Ministry of Education (GOA 2/01), the FWO, and a Max Planck research award are thanked for support.

Supporting Information Available: Details on the setups and techniques used, spectra of the SM depicted in Figure 2, and calculations. This material is available free of charge via the Internet at http://pubs.acs.org.

\section{References}

(1) Tipp, G., Ed. Nanotechnology; Springer: Heidelberg, 1998

(2) Nyberg, T.; Zhang, F.; Inganäs, O. Nanotechnology 2002, 13, 205-211.

(3) Macklin, J. J.; Trautman, J. K.; Harris, T. D.; Brus, L. E. Science 1996, $272,255-258$

(4) Ambrose, W. P.; Goodwin, P. M.; Martin, J. C.; Keller, R. A. Science 1994, 265, 364-367

(5) Ying, L.; Xie, S. J. Phys. Chem. B 1998, 102, 10399-10409.

(6) See Supporting Information.

(7) Drexhage, K. H. J. Lumin. 1970, 1, 693-701.

(8) Vallée, R.; Tomczak, N.; Gersen, H.; van Dijk, E. M. H. P.; García-Parajó, M. F.; Vancso, G. J.; van Hulst, N. F. Chem. Phys. Lett. 2001, 348, 161167.

(9) Enderlein, J. Chem. Phys. 1999, 247, 1-9.

(10) Enderlein, J.; Ruckstuhl, T.; Seeger, S. Appl. Opt. 1999, 38, 724-732.

(11) Chance, R. R.; Prock, A.; Silbey, R. In Advances in Chemical Physics Prigogine, I., Rice, S. R., Eds.; Wiley: New York, 1978; pp 1-65.

(12) Lukosz, W. J. Opt. Soc. Am. 1981, 71, 744-754.

(13) Brorson, S. D. Electromagnetic field mode density calculated via mode counting. In Spontaneous Emission and Laser Oscillations in Microcavities; Yokoyama, H., Ujihara, K., Eds.; CRC Press: Boca Raton, FL, 1995; p 151

14) Bartko, A. P.; Dickson, R. M. J. Phys. Chem. B 1999, 103, 11237-11241.

(15) Sick, B.; Hecht, B.; Novotny, L. Phys. Rev. Lett. 2000, 85, 4482-4485.

(16) Kreiter, M.; Prummer, M.; Hecht, B.; Wild, U. P. J. Chem. Phys. 2002 117, 9430-9433.

(17) Böhmer, M.; Enderlein, J. J. Opt. Soc. Am. B 2003, 20, 554-559.

(18) Hofkens, J.; Vosch, T.; Cotlet, M.; Habuchi, S.; Van Der Biest, K.; Müllen, K.; Dirix, G.; Michiels, J.; Vanderleyden, J.; Sauer, M.; De Schryver, F. C. Proc SPIE 2003, 4962, 1-10.

(19) Morgenroth, F.; Müllen K. Tetrahedron 1997, 53, 15349-15366.

(20) Hofkens, J.; Maus, M.; Gensch, T.; Vosch, T.; Cotlet, M.; Köhn, F.; Herrmann, A.; Müllen, K.; De Schryver, F. C. J. Am. Chem. Soc. 2000 122, 9278-9288.

(21) Patra, D.; Gregor, I.; Enderlein, J. J. Phys. Chem. A, 2004, 108 6836-6841.

JA0474603 